\title{
Interval from transurethral resection of prostate to laparoscopic radical prostatectomy does not affect outcomes for incidental prostate cancer
}

Yu-yong Wang ${ }^{1,2}$, Xiang-yi Zheng' ${ }^{1}$ Qi-qi Mao ${ }^{1}$, Li-ping Xie ${ }^{1}$

\author{
${ }^{1}$ Department of Urology, The First Affiliated Hospital, School of Medicine, Zhejiang \\ University, Hangzhou, China \\ 2Department of Urology, Affiliated Hangzhou First People's Hospital, Zhejiang University \\ School of Medicine, Hangzhou, China
}

Submitted: 14 April 2019

Accepted: 30 October 2019

Arch Med Sci

DOI: https://doi.org/10.5114/aoms.2020.94681

Copyright $\odot 2020$ Termedia \& Banach

\section{Abstract}

Introduction: Laparoscopic radical prostatectomy (LRP) has become a common option for the treatment of prostate cancer. The aim of our study was to examine whether LRP performed within 12 weeks of transurethral resection of the prostate (TURP) is associated with surgical difficulty or outcomes.

Material and methods: A single-institutional retrospective analysis was performed on patients who underwent LRP for incidental prostate cancer after TURP between July 2009 and December 2017. The interval between TURP and LRP was determined and patients with intervals of $\leq 12$ weeks were compared to those with intervals of $>12$ weeks. Patient characteristics, perioperative, pathological, and postoperative functional outcomes were analyzed to determine statistically significant differences between the 2 groups. Multivariable analyses were performed to determine whether the interval between TURP and LRP was a significant independent predictor of these outcomes.

Results: A total of 56 incidental prostate cancer patients detected by TURP were included in this study. No significant differences were detected in estimated blood loss, operative duration, postoperative length of stay, and rate of positive margin, Gleason score upgrading, major complications, incontinence and prostate-specific antigen (PSA) recurrence in patients with a TURP to LRP interval above and below 12 weeks. The TURP to LRP interval was not an independent predictor of outcomes during or after LRP.

Conclusions: Our results showed that performing LRP within 12 weeks after TURP does not adversely influence surgical difficulty or outcomes.

Key words: transurethral resection of prostate, radical prostatectomy, prostatic neoplasms, complications.

\section{Introduction}

Detection of incidental prostate cancer by transurethral resection of the prostate (TURP) has decreased with the introduction of prostatespecific antigen (PSA) screening [1]. Although there is a controversy regarding which treatment is appropriate in cases of incidental detection of prostate cancer, radical prostatectomy (RP) remains a commonly performed treatment $[2,3]$.

It is generally recommended that RP be performed at 12 weeks after TURP, presumably to allow for resolution of inflammatory adhesions

\author{
Corresponding author: \\ Liping Xie \\ Department of Urology \\ The First Affiliated Hospital \\ School of Medicine \\ Zhejiang University \\ Qingchun Road 79 \\ Hangzhou, Zhejiang Province \\ 310003, China \\ E-mail: 22594031@qq.com
}


so that the anatomy between the prostate and the surrounding structures returns to a nearly normal state before surgery. However, there was no convincing data to support this recommendation. During the past decades, laparoscopic radical prostatectomy (LRP) has become a more commonly performed procedure for the treatment of localized prostate cancer [4]. Laparoscopic radical prostatectomy has the advantages of clearer fields of vision, better preservation of anatomical structures, a shorter period of convalescence and less blood loss compared with open RP [5]. To our knowledge, there is a lack of published data whether LRP performed within 12 weeks after TURP was associated with surgical difficulty or outcomes. The present study aims to bridge this gap in the existing literature.

\section{Material and methods}

After obtaining Institutional Review Board approval, we retrospectively reviewed the records of prostate cancer, and included the patients diagnosed at TURP who had undergone LRP between July 2009 and December 2017. We excluded prostate cancer patients detected with prostate biopsy for PSA rising during the post-TURP follow-up, and men who had adjuvant neoadjuvant endocrine therapy were also excluded. The clinical, pathologic and follow-up data were collected to determine whether the interval from TURP to LRP affected the surgical difficulty or outcomes.

We stratified the patients into two groups according to the interval between TURP and LRP: patients with intervals $\leq 12$ weeks and those with intervals > 12 weeks. The age, body mass index (BMI), PSA level before TURP, Gleason score at TURP, operative duration, estimated blood loss $(E B L)$, postoperative length of stay, pathologic Gleason score, complications, Gleason score upgrading, margin status, major complications, postoperative urinary incontinence and PSA recurrence were compared between the 2 groups. Laparoscopic radical prostatectomy was performed by 1 of 2 senior urologists. We used post-operative drop in hemoglobin (preoperative hemoglobin minus post-operative hemoglobin one day after surgery) instead of surgeon-reported estimated blood loss. Patients were considered as having continence if they occasionally leaked a few drops with abdominal straining and if they needed to use only one protective pad a day at 6 months after LRP. Prostate-specific antigen recurrence was defined as follow-up PSA level $>0.2 \mathrm{ng} / \mathrm{ml}$.

The independent $t$-test, $\chi^{2}$ and Fisher's exact test were used to compare the clinical and pathologic variables of patients undergoing LRP across the TURP-to-LRP interval. The multivariate linear regression model was used to examine the effect of the interval between TURP and RP on EBL, operative duration and length of postoperative stay. In addition, we carried out multivariable logistic regression analyses to determine whether the interval from TURP to LRP independently affected the positive surgical margins, Gleason score upgrading, complications, urinary continence and PSA recurrence. We adjusted for potential confounding factors of patient age, BMI, preTURP PSA level, and pathologic Gleason score in the analysis. Statistical analyses were conducted using SPSS 16.0. For all tests, a $p$ value of 0.05 was considered to indicate a significant difference.

\section{Results}

Overall, a total of 56 incidental prostate cancer patients detected by TURP who underwent LRP in our institute were included in this study. The mean age and pre-TURP PSA level were $67.6 \pm 5.7$ and $5.7 \pm 3.6 \mathrm{ng} / \mathrm{ml}$, respectively. Three patients had no residual tumor identifiable in the prostate. Grade of disease ranged from Gleason $3+3=6$ to Gleason $5+4=9$. The positive surgical margin was found in 7 patients. Eight patients developed complications, including pelvic hematoma in one, urine leakage in two, lymphocele formation in three, deep vein thrombosis in one and urethrorectal fistula in one. Forty-five patients were continent at 6 months after RP. Ten patients developed a PSA recurrence, and no patients died during the follow-up.

In all, 38 patients had LRP at $>12$ weeks (17.3 \pm 5.6 weeks) after TURP, and 18 at $\leq 12$ weeks (8.4 \pm 2.5 weeks). There was no significant difference in patients' age, BMI, pre-TURP PSA level, Gleason score at TURP, operative duration, EBL, pathologic Gleason score, length of postoperative stay, rate of Gleason score upgrading, positive margin, complications, urinary incontinence at 6 months after surgery, or PSA recurrence between the $\leq 12$ and $>12$ weeks subgroups (Table I). The TURP to LRP interval was not an independent predictor of EBL, operative duration, length of postoperative stay, positive surgical margins, Gleason score upgrading, complications, urinary incontinence, or PSA recurrence in multivariate linear or logistic regression analysis (Tables II and III). No correlation was found between the TURP to LRP interval and surgical outcomes during or after LRP.

\section{Discussion}

With the introduction of PSA screening, incidental prostate cancer was diagnosed in less than $5 \%$ of patients who undergo benign prostatic hyperplasia-related surgery $[6,7]$. The most appropriate management of incidental prostate cancers diagnosed at TURP has been debated [8]. European Association of Urology guidelines 
Table I. Comparison of preoperative clinical variables, pathologic and follow-up outcomes according to interval between transurethral resection of the prostate (TURP) to laparoscopic radical prostatectomy (LRP)

\begin{tabular}{|c|c|c|c|}
\hline \multirow[t]{2}{*}{ Patients } & \multicolumn{2}{|c|}{ Interval from TURP to LRP (weeks) } & \multirow[t]{2}{*}{$P$ value } \\
\hline & Group A $(\geq 12)$ & Group B $(<12)$ & \\
\hline No. of patients & 38 & 18 & \\
\hline Age [years] & $68.5 \pm 5.9$ & $65.8 \pm 5.0$ & 0.111 \\
\hline BMI $\left[\mathrm{kg} / \mathrm{m}^{2}\right]$ & $23.7 \pm 2.0$ & $25.0 \pm 2.8$ & 0.122 \\
\hline PSA before TURP [ng/ml] & $5.54 \pm 3.64$ & $6.07 \pm 3.52$ & 0.608 \\
\hline Prostate volume [ml] & $31.62 \pm 9.76$ & $33.58 \pm 10.52$ & 0.441 \\
\hline Gleason score at TURP & & & 0.199 \\
\hline 6 & 19 & 14 & \\
\hline $3+4=7$ & 10 & 1 & \\
\hline $4+3=7$ & 4 & 1 & \\
\hline$\geq 8$ & 5 & 2 & \\
\hline Operative duration [min] & $182.3 \pm 54.3$ & $200.2 \pm 64.6$ & 0.284 \\
\hline $\mathrm{EBL}[\mathrm{g} / \mathrm{ll}]$ & $21.4 \pm 12.5$ & $23.4 \pm 15.1$ & 0.59 \\
\hline Pathologic Gleason score & & & 0.618 \\
\hline 6 & 20 & 12 & \\
\hline $3+4=7$ & 9 & 4 & \\
\hline $4+3=7$ & 2 & 0 & \\
\hline$\geq 8$ & 7 & 2 & \\
\hline Gleason score upgrading & 10 & 3 & 0.424 \\
\hline Positive surgical margin & 5 & 2 & 0.829 \\
\hline Complications & 7 & 1 & 0.199 \\
\hline $\begin{array}{l}\text { Postoperative length } \\
\text { of stay [days] }\end{array}$ & $10.7 \pm 6.4$ & $10.8 \pm 3.4$ & 0.954 \\
\hline Urinary incontinence & 8 & 3 & 0.700 \\
\hline PSA recurrence & 6 & 4 & 0.557 \\
\hline
\end{tabular}

$B M I$ - body mass index, TURP - transurethral resection of the prostate, EBL - estimated blood loss, PSA - prostate-specific antigen, $L R P$ - laparoscopic radical prostatectomy.

Table II. Results of the linear regression analysis to test the association between transurethral resection of the prostate to laparoscopic radical prostatectomy interval and outcomes during and after surgery

\begin{tabular}{|lcc|}
\hline Variable & Point estimate $(95 \% \mathrm{Cl})$ & $P$ value \\
\hline EBL $[\mathrm{g} / \mathrm{l}]$ & $-0.15(-7.80,7.50)$ & 0.968 \\
\hline Length of postoperative stay [days] & $-0.97(-4.06,2.12)$ & 0.530 \\
\hline Operative duration [min] & $-13.07(-47.72,21.60)$ & 0.453 \\
\hline
\end{tabular}

EBL - estimated blood loss.

recommend active surveillance for stage T1a and radical prostatectomy for stage $\mathrm{T} 1 \mathrm{~b}$ patients with a life expectancy of more than 10 years [9]. In our study, the mean PSA was higher; hence there was a greater possibility that these incidental prostate cancers would be locally advanced prostate can- cer rather than "true" incidental prostate cancer. As the concept of active surveillance is not widely accepted in China, most patients with incidental prostate cancer after TURP will undergo RP.

Many studies have described the procedure as being more difficult after TURP [10]. Perforation 
Table III. Results of the multivariate logistic regression analysis to test for associations between transurethral resection of the prostate to laparoscopic radical prostatectomy interval and postoperative outcomes

\begin{tabular}{|lcc|}
\hline Variable & Odds ratio $(95 \% \mathrm{Cl})$ & $P$ value \\
\hline $\begin{array}{l}\text { Positive surgical } \\
\text { margins }\end{array}$ & $1.469(0.20-11.03)$ & 0.709 \\
\hline $\begin{array}{l}\text { Gleason score } \\
\text { upgrading }\end{array}$ & $1.30(0.25-6.65)$ & 0.759 \\
\hline Urinary incontinence & $1.16(0.24-5.59)$ & 0.857 \\
\hline Complications & $3.32(0.27-41.6)$ & 0.352 \\
\hline PSA recurrence & $0.68(0.13-3.50)$ & 0.645 \\
\hline
\end{tabular}

of the prostatic capsule during TURP with extravasation of blood and irrigation fluid can lead to periprostatic fibrosis and distortion of the surgical planes. Bladder neck sparing can seldom be performed, and neurovascular bundle (NVB) preservation is technically more difficult, ultimately compromising postoperative continence and erectile function [11]. In the era of open RP, the TURP to LRP interval has been recommended as at least 12 weeks to allow adequate time for complete resolution of inflammation [12]. However, the reason for this recommendation is not yet clear. It may originate from clinical experience of urologists with challenging cases after a short interval to TURP. In the last decades, LRP has become a common option for the treatment of prostate cancer and represents a new experience for urologists. However, tissue adhesion is also considered to be one of the factors that make the laparoscopic surgery more difficult [13]. In actual clinical practice, urologists often encounter patients who choose RP and request surgery as soon as possible. For such patients, our results showed that LRP performed within 12 weeks did not affect surgical difficulty, such as operative duration, EBL and major complications, suggesting that patients could safely proceed to LRP shortly after TURP. Although insignificant, we noted that the complications rate in patients with an interval below 12 weeks was obviously higher than in those with an interval above 12 weeks ( $18.4 \%$ vs. $5.6 \%$ ). It could be speculated that this difference might become significant if more patients were enrolled.

Because such tumors are often perceived to be of low volume and clinically insignificant, and several therapeutic options are available, some patients would need a long time to finalize their decision when choosing a primary treatment for incidental prostate cancer. Our results indicated no significant differences in rate of positive margin, Gleason score upgrading, urinary incontinence or PSA recurrence in patients with TURP to
RP intervals above and below 12 weeks, suggesting that patients choosing delayed treatment were not at risk of disease progression and the surgical efficacy was not affected by a longer TURP to LRP interval.

To the best of our knowledge, this is the first study comparing incidental prostate cancer patients of TURP-to-LRP interval $\leq 12$ weeks with those $>12$ weeks. Although all patients had LRP in this analysis, these findings may be applicable to open RP or robotic-assisted LRP. As with TURP, it is a widely accepted practice to wait at least 6 weeks after prostate biopsy before RP to allow for resolution of inflammation and bleeding caused by biopsy [14]. However, consistent with the present study, previous work also showed no significant association between biopsy-to-surgery interval and perioperative outcomes $[15,16]$.

There are several limitations to our study that warrant discussion. First, our study had a relatively small sample size, especially for the patients with an interval below 12 weeks. More patients need to be included to improve the statistical power. Second, it is retrospective in nature, so there may be confounding variables that are unaccounted for. A prospective analysis with different intervals can allow for a better definition of a minimum and an optimum TURP to LRP interval. Third, our data are from a single institution. Some of our findings might be specific to surgical techniques or patient selection, and therefore may have less applicability elsewhere. Last, the post-operative erectile function was not analyzed, as we did not preserve the NVBs intraoperatively, and that would require a longer follow-up and standardized instruments, which were not used to assess erectile function in these patients.

In conclusion, our study showed that TURPto-LRP interval did not affect surgical difficulty or outcomes. This may provide reassurance for either the urologists or patients contemplating LRP soon after TURP. However, our results need to be further confirmed in large prospective studies.

\section{Acknowledgments}

This study is supported by grants from the Zhejiang Provincial Medical Science Foundation of China (2013KYB086) and the Natural Science Foundation of Zhejiang Province (LY18H160010).

\section{Conflict of interest}

The authors declare that they have no conflict of interest.

\section{References}

1. Tombal B, De Visccher L, Cosyns JP, et al. Assessing the risk of unsuspected prostate cancer in patients with benign prostatic hypertrophy: a 13-year retrospective 
study of the incidence and natural history of T1a-T1b prostate cancers. BJU Int 1999; 84: 1015-20.

2. Fragkoulis C, Pappas A, Theocharis G, Papadopoulos G, Stathouros G, Ntoumas K. Open radical prostatectomy after transurethral resection: perioperative, functional, oncologic outcomes. Canadian J Urol 2018; 25: 9262-7.

3. Yang Y, Luo Y, Hou GL, et al. Laparoscopic radical prostatectomy after previous transurethral resection of the prostate in clinical $\mathrm{T} 1 \mathrm{a}$ and $\mathrm{T} 1 \mathrm{~b}$ prostate cancer: a matched-pair analysis. Urol J 2015; 12: 2154-9.

4. Brassetti A, Bollens R. Laparoscopic radical prostatectomy in 2018: 20 years of worldwide experiences, experimentations, researches and refinements. Minerva Chir 2019; 74: 37-53.

5. Zugor V, Labanaris AP, Porres D, Witt JH. Surgical, oncologic, and short-term functional outcomes in patients undergoing robot-assisted prostatectomy after previous transurethral resection of the prostate. J Endourol 2012; 26: 515-9.

6. Capitanio U. Contemporary management of patients with T1a and T1b prostate cancer. Curr Opin Urol 2011; 21: 252-6.

7. Zigeuner RE, Lipsky K, Riedler I, et al. Did the rate of incidental prostate cancer change in the era of PSA testing? A retrospective study of 1127 patients. Urology 2003; 62: 451-5.

8. Lee DH, Chung DY, Lee KS, et al. Clinical experiences of incidental prostate cancer after transurethral resection of prostate (TURP) according to initial treatment: a study of a Korean high volume center. Yonsei Medical Journal 2014; 55: 78-83.

9. Heidenreich A, Bellmunt J, Bolla M, et al. EAU guidelines on prostate cancer. Part 1: screening, diagnosis, and treatment of clinically localised disease. Eur Urol 2011; 59: 61-71.

10. Verze P, Greco F, Scuzzarella S, et al. The impact of previous prostate surgery on the outcomes of laparoscopic radical prostatectomy. Minerva Urol Nefrol 2017; 69: 76-84.

11. Bratu O, Spînu D, Oprea I, et al. Complications of radical retropubic prostatectomy-our experience. Romanian Journal of Military Medicine 2015; 118: 23-25.

12. Wein A, Kavoussi L, Partin A, Peters C. Campbell-Walsh Urology. $17^{\text {th }}$ ed. Philadelphia: Elsevier; 2016, p. 2644.

13. Davey AK, Maher PJ. Surgical adhesions: a timely update, a great challenge for the future. J Minim Invasive Gynecol 2007; 14: 15-22.

14. Sokoloff MH, Brendler CB. Indications and contraindications for nerve-sparing radical prostatectomy. Urol Clin North Am 2001; 28: 535-43.

15. Hirasawa $Y$, Ohori $M$, Sugihara T, et al. No clinical significance of the time interval between biopsy and roboticassisted radical prostatectomy for patients with clinically localized prostate cancer on biochemical recurrence: a propensity score matching analysis. Jpn J Clin Oncol 2017; 47: 1083-9.

16. Park B, Choo SH, Jeon HG, et al. Interval from prostate biopsy to radical prostatectomy does not affect immediate operative outcomes for open or minimally invasive approach. J Korean Med Sci 2014; 29: 1688-93. 